Article

\title{
Applications of Coupled Fixed Points for Multivalued Maps in the Equilibrium in Duopoly Markets and in Aquatic Ecosystems
}

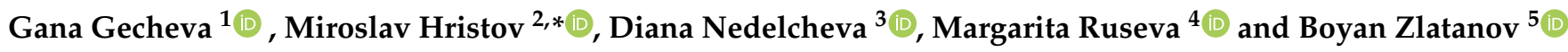 \\ 1 Department of Ecology and Environmental Conservation, Faculty of Biology, University of Plovdiv "Paisii \\ Hilendarski", 24 Tzar Assen Str., 4000 Plovdiv, Bulgaria; ggecheva@uni-plovdiv.bg \\ 2 Department of Algebra and Geometry, Faculty of Mathematics and Computer Science, University of Shumen \\ "Konstantin Preslavsky", 115 Universitetska Str., 9700 Shumen, Bulgaria \\ 3 Department of Mathematics and Physics, Faculty of Electrical Engineering, Technical University of Varna, 1 \\ Studentska Str., 9000 Varna, Bulgaria; diana.nedelcheva@tu-varna.bg \\ 4 Department of Management and Quantitative Economicsx, Faculty of Economics and Social Sciences, \\ University of Plovdiv "Paisii Hilendarski", 24 Tzar Assen Str., 4000 Plovdiv, Bulgaria; \\ m_ruseva@uni-plovdiv.bg \\ 5 Department of Mathematical Analysis, Faculty of Mathematics and Informatics, University of Plovdiv “Paisii \\ Hilendarski", 24 Tzar Assen Str., 4000 Plovdiv, Bulgaria; bobbyz@uni-plovdiv.bg \\ * Correspondence: miroslav.hristov@shu.bg; Tel.: +359-89-336-5666
}

check for

updates

Citation: Gecheva, G.; Hristov, M.;

Nedelcheva, D.; Ruseva, M.; Zlatanov,

B. Applications of Coupled Fixed

Points for Multivalued Maps in the

Equilibrium in Duopoly Markets and

in Aquatic Ecosystems. Axioms 2021,

10, 44. https://doi.org/10.3390/

axioms 10020044

Academic Editor: Wei-Shih Du

Received: 25 February 2021

Accepted: 22 March 2021

Published: 26 March 2021

Publisher's Note: MDPI stays neutral with regard to jurisdictional claims in published maps and institutional affiliations.

Copyright: (c) 2021 by the authors. Licensee MDPI, Basel, Switzerland. This article is an open access article distributed under the terms and conditions of the Creative Commons Attribution (CC BY) license (https:// creativecommons.org/licenses/by/ $4.0 /)$.
Abstract: We have obtained a new class of ordered pairs of multivalued maps that have pairs of coupled fixed points. We illustrate the main result with two examples that cover a wide range of models. We apply the main result in models in duopoly markets to get a market equilibrium and in aquatic ecosystems, also to get an equilibrium.

Keywords: multivalued maps; coupled fixed points; equilibrium

MSC: Primary 47H04; 47H10; 91B26

\section{Introduction}

Fixed point theory has been extensively researched and widely applied in a multitude of directions for many years. The "Banach Contraction Principle" states that under certain conditions a self map $T$ on a set $X$ admits one or more fixed points $x=T x$. The "Banach Contraction Principle" and its numerous generalizations are widely used in many branches of mathematics because it requires only the structure of a complete metric space with conditions on the map which are easily tested.

We will mention just a few directions of the generalizations (fixed points for set-valued maps, coupled fixed points, fixed points for cyclic maps) of "Banach Contraction Principle" that initiate the present investigation.

Following the "Banach Contraction Principle", Nadler introduced the concept of set-valued contractions in [1]. He also proved that a set-valued contraction possesses a fixed point in a complete metric space. In the late twentieth century Dontchev and Hager successfully presented an extension of Nadler's result in [2]. They determined the location of a fixed point with respect to an initial value of the set-valued mapping. Their conclusion was obtained under two modified conditions and it has since been playing an important role in the development of the metric fixed point theory. We would like just to mention a few recent results about fixed points for set-valued maps and their applications [3-6].

A different direction is the notion of coupled fixed points introduced in [7]. There are a lot of recent results about coupled fixed points [8-11]. 
Another kind of generalization of the Banach contraction principle is the notation of cyclic maps [12] and later its generalization to the best proximity point, introduced in [13]. The definition presented in [13] is more general than the one in [12], in the sense that if the sets intersect, then every best proximity point is a fixed point. Some very recent results in this field are presented in [14-17].

It seems that recently all mentioned directions of research in fixed point theory are of interest.

By combining the notions of coupled fixed (or best proximity) points for cyclic maps, a model of duopoly market was built in $[18,19]$.

We will try to enrich the notions of set-valued maps, coupled fixed points, cyclic maps and to get results that we will apply in economics and ecology.

\section{Preliminaries}

We will recall basic notions and facts that we will need for investigation of coupled fixed points for multi-valued maps. Let $(X, \rho)$ and $(Y, \sigma)$ be two metric spaces. We will denote by $B_{X, r}(\bar{x})$ the open ball and by $B_{X, r}[\bar{x}]$ the closed ball with a radius $r$ and a center $x$ in the metric space $X$. If no confusion arises, we will denote them with $B_{r}(\bar{x})$ and $B_{r}[\bar{x}]$, respectively. Let $x \in X$ and $C \subset X$. We will denote the distance from $x$ to $C$ by $d(x, C)=\inf \{\rho(x, z): z \in C\}$. If $C=\varnothing$ then we put $d(x, \varnothing)=\infty$.

Let $A, B \subset X$ be two subsets. An excess of $A$ beyond $B$ is called $e(A, B)=\sup \{d(x, B)$ : $x \in A\}$, where the convention is used that

$$
e(\varnothing, B)=\left\{\begin{array}{cc}
0, & B \neq \varnothing \\
\infty, & B=\varnothing
\end{array} .\right.
$$

Let $(X, \rho)$ and $(Y, \sigma)$ be two metric spaces. Let us denote by $F: X \rightrightarrows Y$ a set-valued mapping defined on the metric space $(X, \rho)$ with values in the metric space $(Y, \sigma)$. Let $F$ be a set-valued map: Its graph is the set gph $F=\{(x, y) \in X \times Y \mid y \in F(x)\}$, its effective domain is the set $\operatorname{dom} F=\{x \in X \mid F(x) \neq \varnothing\}$ and its effective range is $\operatorname{rge} F=\{y \in Y \mid$ there exists $x$ such that $y \in F(x)\}$.

Definition 1. ([1]) A point $x \in X$ is said to be a fixed point of the set-valued map $F: X \rightrightarrows X$ if $x \in F(x)$.

Definition 2. ([7]) A point $(x, y) \in X \times X$ is said to be a coupled fixed point of the map $F$ : $X \times X \rightarrow X$ if $x=F(x, y)$ and $y=F(y, x)$.

Definition 3. ([20]) A point $(x, y) \in X \times X$ is said to be a coupled fixed point of the set-valued map $F: X \times X \rightrightarrows X$ if $x \in F(x, y)$ and $y \in F(y, x)$.

The model that will be constructed in the application section will be of two set-valued maps $F_{1}: X \times Y \rightrightarrows X$ and $F_{2}: X \times Y \rightrightarrows Y$ and we will be interested in the existence of ordered pairs $(x, y)$, such that $x \in F_{1}(x, y)$ and $y \in F_{2}(x, y)$, which are called generalized coupled fixed points for the ordered pair of set-valued maps $\left(F_{1}, F_{2}\right)$.

\section{Main Results}

We will present a result, which extends the result from [2] and establishes a solution of the generalized coupled fixed point problem for an ordered pair of set-valued maps.

Theorem 1. Let $(X, \rho)$ and $(Y, \sigma)$ be complete metric spaces, $F_{1}: X \times Y \rightrightarrows X$ and $F_{2}: X \times Y \rightrightarrows$ $Y$ be multi-valued maps and $\bar{x} \in X, \bar{y} \in Y$. Let there exist $a$ constant $r>0$ and non-negative constants $\alpha, \beta, \gamma, \delta$, satisfying $\max \{\alpha+\gamma, \beta+\delta\}<1$, such that the following assumptions hold:

(a) $F_{1}(x, y)$ and $F_{2}(x, y)$ are nonempty closed subsets of $X$ and $Y$ for all $(x, y) \in B_{r}(\bar{x}) \times B_{r}(\bar{y})$

(b) the inequality $d\left(\bar{x}, F_{1}(\bar{x}, \bar{y})\right)+d\left(\bar{y}, F_{2}(\bar{x}, \bar{y})\right)<r(1-\lambda)$ holds, where $\lambda=\max \{\alpha+\gamma, \beta+\delta\}$ 
(c) the inequality

$$
\begin{aligned}
S_{1} & =e\left(F_{1}(x, y) \cap B_{r}(\bar{x}), F_{1}(u, v)\right)+e\left(F_{2}(z, w) \cap B_{r}(\bar{y}), F_{2}(t, s)\right) \\
& \leq \alpha \rho(x, u)+\beta \sigma(y, v)+\gamma \rho(z, t)+\delta \sigma(w, s)
\end{aligned}
$$

holds for all $(x, y),(u, v),(z, w),(t, s) \in B_{r}(\bar{x}) \times B_{r}(\bar{y})$.

Then, the generalized coupled fixed point problem has at least one solution $(x, y) \in B_{r}(\bar{x}) \times B_{r}(\bar{y})$.

Proof. Let $\bar{x}=x_{0}$ and $\bar{y}=y_{0}$.

We will construct by induction a sequence $\left\{\left(x_{n}, y_{n}\right)\right\}_{n=1}^{\infty}$, which will satisfy the inclusions $x_{n+1} \in F_{1}\left(x_{n}, y_{n}\right) \cap B_{r}\left(x_{0}\right)$ and $y_{n+1} \in F_{2}\left(x_{n}, y_{n}\right) \cap B_{r}\left(y_{0}\right)$ for $n \geq 0$.

Step one of the induction: We will choose $\left(x_{1}, y_{1}\right)$. By assumption $(b)$, there exist $x_{1} \in F_{1}\left(x_{0}, y_{0}\right)$ and $y_{1} \in F_{2}\left(x_{0}, y_{0}\right)$ such that $\rho\left(x_{1}, x_{0}\right)+\sigma\left(y_{1}, y_{0}\right)<r(1-\lambda)<r$. Therefore, $x_{1} \in F_{1}\left(x_{0}, y_{0}\right) \cap B_{r}\left(x_{0}\right)$ and $y_{1} \in F_{2}\left(x_{0}, y_{0}\right) \cap B_{r}\left(y_{0}\right)$.

We will proceed with the choice of $\left(x_{2}, y_{2}\right)$. From (1) we have the chain of inequalities

$$
\begin{aligned}
S_{2} & =d\left(x_{1}, F_{1}\left(x_{1}, y_{1}\right)\right)+d\left(y_{1}, F_{2}\left(x_{1}, y_{1}\right)\right) \\
& \leq e\left(F_{1}\left(x_{0}, y_{0}\right) \cap B_{r}\left(x_{0}\right), F_{1}\left(x_{1}, y_{1}\right)\right)+e\left(F_{2}\left(x_{0}, y_{0}\right) \cap B_{r}\left(y_{0}\right), F_{2}\left(x_{1}, y_{1}\right)\right) \\
& \leq \alpha \rho\left(x_{1}, x_{0}\right)+\beta \sigma\left(y_{1}, y_{0}\right)+\gamma \rho\left(x_{1}, x_{0}\right)+\delta \sigma\left(y_{1}, y_{0}\right) \\
& =(\alpha+\gamma) \rho\left(x_{1}, x_{0}\right)+(\beta+\delta) \sigma\left(y_{1}, y_{0}\right) \\
& \leq \max \{\alpha+\gamma, \beta+\delta\}\left(\rho\left(x_{1}, x_{0}\right)+\sigma\left(y_{1}, y_{0}\right)\right) \\
& <r(1-\lambda) \lambda .
\end{aligned}
$$

The above inequalities imply the existence of $x_{2} \in F_{1}\left(x_{1}, y_{1}\right)$ and $y_{2} \in F_{2}\left(x_{1}, y_{1}\right)$, such that $\rho\left(x_{2}, x_{1}\right)+\sigma\left(y_{2}, y_{1}\right)<r(1-\lambda) \lambda$. Using the triangular inequality we get

$$
\begin{aligned}
\rho\left(x_{2}, x_{0}\right)+\sigma\left(y_{2}, y_{0}\right) & \leq \rho\left(x_{2}, x_{1}\right)+\sigma\left(y_{2}, y_{1}\right)+\rho\left(x_{1}, x_{0}\right)+\sigma\left(y_{1}, y_{0}\right) \\
& \leq r(1-\lambda) \lambda+r(1-\lambda)<r .
\end{aligned}
$$

Consequently $x_{2} \in F_{1}\left(x_{1}, y_{1}\right) \cap B_{r}\left(x_{0}\right)$ and $y_{2} \in F_{2}\left(x_{1}, y_{1}\right) \cap B_{r}\left(y_{0}\right)$.

Step two of the induction: Let us suppose that we have already chosen $\left\{\left(x_{k}, y_{k}\right)\right\}_{k=1}^{n}$, satisfying for each $k=1,2, \ldots, n$

$$
x_{k} \in F_{1}\left(x_{k-1}, y_{k-1}\right) \cap B_{r}\left(x_{0}\right), y_{k} \in F_{2}\left(x_{k-1}, y_{k-1}\right) \cap B_{r}\left(y_{0}\right)
$$

and

$$
\rho\left(x_{k}, x_{k-1}\right)+\sigma\left(y_{k}, y_{k-1}\right)<r(1-\lambda) \lambda^{k-1} .
$$

Step three of the induction: We will prove that we can choose $\left(x_{n+1}, y_{n+1}\right)$, provided that we have already chosen $\left\{\left(x_{k}, y_{k}\right)\right\}_{k=1}^{n}$. By assumption (1) we have the chain of inequalities

$$
\begin{aligned}
S_{3} & =d\left(x_{n}, F_{1}\left(x_{n}, y_{n}\right)\right)+d\left(y_{n}, F_{2}\left(x_{n}, y_{n}\right)\right) \\
& \leq e\left(F_{1}\left(x_{n-1}, y_{n-1}\right) \cap B_{r}\left(x_{0}\right), F_{1}\left(x_{n}, y_{n}\right)\right)+e\left(F_{2}\left(x_{n-1}, y_{n-1}\right) \cap B_{r}\left(y_{0}\right), F_{2}\left(x_{n}, y_{n}\right)\right) \\
& \leq \alpha \rho\left(x_{n}, x_{n-1}\right)+\beta \sigma\left(y_{n}, y_{n-1}\right)+\gamma \rho\left(x_{n}, x_{n-1}\right)+\delta \sigma\left(y_{n}, y_{n-1}\right) \\
& =(\alpha+\gamma) \rho\left(x_{n}, x_{n-1}\right)+(\beta+\delta) \sigma\left(y_{n}, y_{n-1}\right) \\
& \leq \max \{\alpha+\gamma, \beta+\delta\}\left(\rho\left(x_{n}, x_{n-1}\right)+\sigma\left(y_{n}, y_{n-1}\right)\right) \\
& <\lambda r(1-\lambda) \lambda^{n-1}=r(1-\lambda) \lambda^{n} .
\end{aligned}
$$

The above inequalities imply that there exist

$$
x_{n+1} \in F_{1}\left(x_{n}, y_{n}\right) \text { and } y_{n+1} \in F_{2}\left(x_{n}, y_{n}\right) \text {, }
$$


such that

$$
\rho\left(x_{n}, x_{n+1}\right)+\sigma\left(y_{n}, y_{n+1}\right)<r(1-\lambda) \lambda^{n} .
$$

Using the triangular inequality we get

$$
\rho\left(x_{n+1}, x_{0}\right)+\sigma\left(y_{n+1}, y_{0}\right) \leq \sum_{k=0}^{n} \rho\left(x_{k+1}, x_{k}\right)+\sigma\left(y_{k+1}, y_{k}\right)<r(1-\lambda) \sum_{k=0}^{n} \lambda^{k}<r .
$$

Consequently, $x_{n+1} \in F_{1}\left(x_{n}, y_{n}\right) \cap B_{r}\left(x_{0}\right)$ and $y_{n+1} \in F_{2}\left(x_{n}, y_{n}\right) \cap B_{r}\left(y_{0}\right)$ and this completes the induction.

It follows from (2) that $\max \left\{\rho\left(x_{n}, x_{n+1}\right), \sigma\left(y_{n}, y_{n+1}\right)\right\}<r(1-\lambda) \lambda^{n}$ and thus the inequality

$$
\rho\left(x_{n}, x_{m}\right) \leq \sum_{k=m}^{n-1} \rho\left(x_{k+1}, x_{k}\right)<r(1-\lambda) \lambda^{m} \sum_{k=0}^{n-1} \lambda^{k}<r \lambda^{m}
$$

holds for any $n>m$.

Therefore, $\left\{x_{n}\right\}_{n=0}^{\infty}$ is a Cauchy sequence and from the assumption that $X$ is a complete metric space it follows that $\left\{x_{n}\right\}_{n=0}^{\infty}$ converges to some $x^{*} \in B_{r}\left(x_{0}\right)$. By similar arguments we get that the sequence $\left\{y_{n}\right\}_{n=0}^{\infty}$ is a Cauchy sequence and converges to some $y^{*} \in B_{r}\left(y_{0}\right)$.

By using of assumption (1) we get the chain of inequalities

$$
\begin{aligned}
S_{4} & =d\left(x_{n}, F_{1}\left(x^{*}, y^{*}\right)\right)+d\left(y_{n}, F_{2}\left(x^{*}, y^{*}\right)\right) \\
& \leq e\left(F_{1}\left(x_{n-1}, y_{n-1}\right) \cap B_{r}\left(x_{0}\right), F_{1}\left(x^{*}, y^{*}\right)\right)+e\left(F_{2}\left(x_{n-1}, y_{n-1}\right) \cap B_{r}\left(y_{0}\right), F_{2}\left(x^{*}, y^{*}\right)\right) \\
& \leq \alpha \rho\left(x^{*}, x_{n-1}\right)+\beta \sigma\left(y^{*}, y_{n-1}\right)+\gamma \rho\left(x^{*}, x_{n-1}\right)+\delta \sigma\left(y^{*}, y_{n-1}\right) \\
& \leq(\alpha+\gamma) \rho\left(x^{*}, x_{n-1}\right)+(\beta+\delta) \sigma\left(y^{*}, y_{n-1}\right) \\
& \leq \lambda\left(\rho\left(x^{*}, x_{n-1}\right)+\sigma\left(y^{*}, y_{n-1}\right)\right) .
\end{aligned}
$$

Applying the triangle inequality we obtain

$$
\begin{aligned}
S_{5} & =d\left(x^{*}, F_{1}\left(x^{*}, y^{*}\right)\right)+d\left(y^{*}, F_{2}\left(x^{*}, y^{*}\right)\right) \\
& \leq \rho\left(x^{*}, x_{n}\right)+d\left(x_{n}, F_{1}\left(x^{*}, y^{*}\right)\right)+\sigma\left(y^{*}, y_{n}\right)+d\left(y_{n}, F_{2}\left(x^{*}, y^{*}\right)\right) \\
& \leq \rho\left(x^{*}, x_{n}\right)+\lambda\left(\left(\rho\left(x^{*}, x_{n-1}\right)+\sigma\left(y^{*}, y_{n-1}\right)\right)+\sigma\left(y^{*}, y_{n}\right) .\right.
\end{aligned}
$$

After taking a limit as $n \rightarrow \infty$ in (3) we get that $d\left(x^{*}, F_{1}\left(x^{*}, y^{*}\right)\right)+d\left(y^{*}, F_{2}\left(x^{*}, y^{*}\right)\right)=0$. From the assumption that $F_{1}\left(x^{*}, y^{*}\right)$ and $F_{2}\left(x^{*}, y^{*}\right)$ are closed it follows that $x^{*} \in F_{1}\left(x^{*}, y^{*}\right)$ and $y^{*} \in F_{2}\left(x^{*}, y^{*}\right)$, i.e $\left(x^{*}, y^{*}\right)$ is a generalized coupled fixed point.

Remark 1. If $F_{1}$ and $F_{2}$ are single-valued, then assumption (1) implies that $\left(x^{*}, y^{*}\right)$ is the unique coupled fixed point of $\left(F_{1}, F_{2}\right)$ in $B_{r}\left(x_{0}\right) \times B_{r}\left(y_{0}\right)$.

\section{Examples and Applications}

We will illustrate Theorem 1 with two examples. We will use these two examples to construct models in economics and ecology.

\subsection{Examples}

Example 1: Let us choose $0 \leq \alpha<\beta<\gamma<\delta \leq \eta<+\infty, n, m \in(0,1]$, so that

$$
\max \left\{\frac{n(\gamma-\beta)+m(\gamma-\beta)}{2\left((\eta+1)^{n}-(\alpha+1)^{n}\right)}, \frac{n(\gamma-\beta)+m(\gamma-\beta)}{2(\delta+1)^{n}}\right\}<1 .
$$

Let us define the maps

$$
f:[0, \delta] \rightarrow\left[\frac{\beta+\gamma}{2}, \gamma\right], g:[\alpha, \eta] \rightarrow\left[\beta, \frac{\beta+\gamma}{2}\right],
$$




$$
\varphi:[0, \delta] \rightarrow\left[\frac{\beta+\gamma}{2}, \gamma\right], \psi:[\alpha, \eta] \rightarrow\left[\beta, \frac{\beta+\gamma}{2}\right]
$$

by

$$
\begin{gathered}
f(x)=\frac{\gamma-\beta}{2(\delta+1)^{n}}(x+1)^{n}+\frac{\beta+\gamma}{2}, \\
g(x)=\frac{\gamma-\beta}{2\left((\eta+1)^{n}-(\alpha+1)^{n}\right)}(x+1)^{n}+\beta-(\alpha+1)^{n} \frac{\gamma-\beta}{2\left((\eta+1)^{n}-(\alpha+1)^{n}\right)}, \\
\varphi(x)=\frac{\gamma-\beta}{2(\delta+1)^{m}}(x+1)^{m}+\frac{\beta+\gamma}{2}, \\
\psi(x)=\frac{\gamma-\beta}{2\left((\eta+1)^{m}-(\alpha+1)^{m}\right)}(x+1)^{m}+\beta-(\alpha+1)^{m} \frac{\gamma-\beta}{2\left((\eta+1)^{m}-(\alpha+1)^{m}\right)} .
\end{gathered}
$$

Let us denote $\bar{x}=\bar{y}=\frac{\beta+\gamma}{2}$ and $\theta=\min \{|\delta-\bar{x}|,|\alpha-\bar{x}|\}$. Let us endow $\mathbb{R}$ with the absolute value metrics $|\cdot-\cdot|$. Let us consider the sets $X=[0, \delta], Y=[\alpha, \eta]$. Let us define the multivalued maps $F: X \times Y \rightrightarrows X$ and $G: X \times Y \rightrightarrows Y$ by

$$
F(x, y)=\{\xi: g(y) \leq \xi \leq f(x)\}
$$

and

$$
G(x, y)=\{\xi: \psi(y) \leq \xi \leq \varphi(x)\} .
$$

We will check that $F$ and $G$ satisfy Theorem 1 .

It is easy to see that for any $(x, y) \in B_{r}(\bar{x}) \times B_{r}(\bar{y})$ the sets $F(x, y)=[g(y), f(x)]$ and $G(x, y)=[\psi(y), \varphi(x)]$ are non empty and closed subsets of $X$ or $Y$, respectively.

From $g(\bar{x}) \leq \bar{x} \leq f(\bar{x})$ and $\psi(\bar{x}) \leq \bar{x} \leq \varphi(\bar{x})$ we get that $F(\bar{x}, \bar{x}) \rightrightarrows[g(\bar{x}), f(\bar{x})]$ and $G(\bar{x}, \bar{x}) \rightrightarrows[\psi(\bar{x}), \varphi(\bar{x})]$. Then

$$
d(\bar{x}, F(\bar{x}, \bar{x}))+d(\bar{x}, G(\bar{x}, \bar{x}))=0<r(1-\lambda)
$$

for any $r>0$ and any $\lambda \in[0,1)$.

From $F(x, y) \rightrightarrows[g(y), f(x)] \subseteq B_{r}(\bar{x})$ and $G(x, y) \rightrightarrows[\psi(y), \varphi(x)] \subseteq B_{r}(\bar{y})$ it follows that $F(x, y) \cap B_{r}(\bar{x})=F(x, y)=[g(y), f(x)]$ and $G(x, y) \cap B_{r}(\bar{y})=G(x, y)=[\psi(y), \varphi(x)]$ for $r=\theta$.

Consequently,

$$
\begin{aligned}
e\left(F(x, y) \cap B_{r}(\bar{x}), F(u, v)\right) & =e([g(y), f(x)],[g(v), f(u)]) \\
& =\sup _{x \in[g(y), f(x)]} d(x,[g(v), f(u)]) .
\end{aligned}
$$

There are four cases:

(I) $x \leq u$ and $y \leq v$; (II) $x \leq u$ and $y \geq v$; (III) $x \geq u$ and $y \leq v$; (IV) $x \geq u$ and $y \geq v$. We will need the inequalities:

$$
|f(x)-f(y)|=f^{\prime}(\zeta)|x-y| \leq n \frac{\gamma-\beta}{2\left((\eta+1)^{n}-(\alpha+1)^{n}\right)}|x-y|,
$$

and

$$
|g(x)-g(y)|=g^{\prime}(\zeta)|x-y| \leq n \frac{\gamma-\beta}{2(\delta+1)^{n}}|x-y| .
$$

Case (I). We will illustrate this case with a figure for easier reading (Figure 1). The other three cases are similar. 


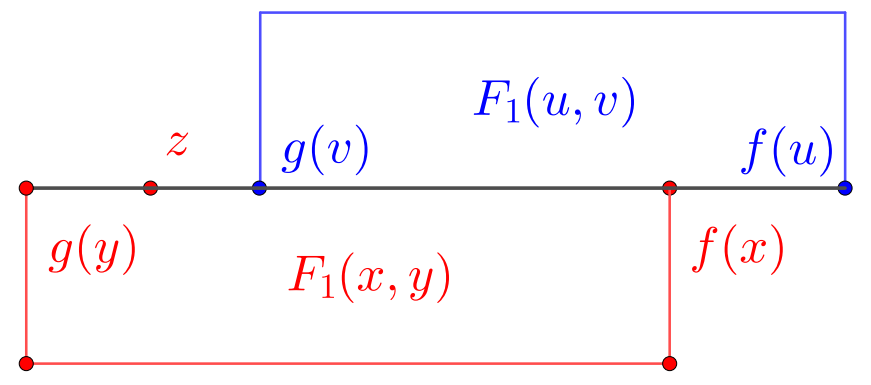

Figure 1. Case (I) $x \leq u$ and $y \leq v$.

$$
\begin{aligned}
S_{5} & =\sup _{z \in[g(y), f(x)]} d(z,[g(v), f(u)]) \\
& =\sup _{g(y) \leq z \leq g(v)}|z-g(v)|=|g(y)-g(v)|=n \frac{\gamma-\beta}{2(\delta+1)^{n}}|y-v| .
\end{aligned}
$$

Case (II). In this case $F_{1}(x, y) \subseteq F_{1}(u, v)$ and we get

$$
\sup _{z \in[g(y), f(x)]} d(z,[g(v), f(u)])=0 .
$$

Case (III). In this case $F_{1}(u, v) \subseteq F_{1}(x, y)$ and we get

$$
\begin{aligned}
S_{6} & =\sup _{z \in[g(y), f(x)]} d(z,[g(v), f(u)])=\max \{|g(y)-g(v)|,|f(x)-f(u)|\} \\
& =\max \left\{n \frac{\gamma-\beta}{2(\delta+1)^{n}}|y-v|, n \frac{\gamma-\beta}{2\left((\eta+1)^{n}-(\alpha+1)^{n}\right)}|x-u|\right\} .
\end{aligned}
$$

Case (IV). This case is very similar to case I) and we get

$$
\sup _{z \in[g(y), f(x)]} d(z,[g(v), f(u)])=|f(x)-f(u)|=n \frac{\gamma-\beta}{2\left((\eta+1)^{n}-(\alpha+1)^{n}\right)}|x-u| .
$$

Therefore by combining the four Cases (I) to (IV) we get

$$
\begin{aligned}
S_{7} & =e\left(F(x, y) \cap B_{r}(\bar{x}), F(u, v)\right) \\
& \leq \max \left\{n \frac{\gamma-\beta}{2(\delta+1)^{n}}|y-v|, n \frac{\gamma-\beta}{2\left((\eta+1)^{n}-(\alpha+1)^{n}\right)}|x-u|\right\} \\
& \leq n \frac{\gamma-\beta}{2(\delta+1)^{n}}|y-v|+n \frac{\gamma-\beta}{2\left((\eta+1)^{n}-(\alpha+1)^{n}\right)}|x-u| .
\end{aligned}
$$

By similar calculations we can get that

$$
\begin{aligned}
S_{8} & =e\left(G(z, w) \cap B_{r}(\bar{y}), G(t, s)\right) \\
& \leq m \frac{\gamma-\beta}{2(\delta+1)^{m}}|w-s|+m \frac{\gamma-\beta}{2\left((\eta+1)^{m}-(\alpha+1)^{m}\right)}|z-t| .
\end{aligned}
$$

Thus there holds the inequality

$$
\begin{aligned}
S_{9}= & e\left(F(x, y) \cap B_{r}(\bar{x}), F(u, v)\right)+e\left(G(z, w) \cap B_{r}(\bar{y}), G(t, s)\right) \\
\leq & n \frac{(\gamma-\beta)|x-u|}{2\left((\eta+1)^{n}-(\alpha+1)^{n}\right)}+m \frac{(\gamma-\beta)|z-t|}{2\left((\eta+1)^{m}-(\alpha+1)^{m}\right)} \\
& +n \frac{\gamma-\beta}{2(\delta+1)^{n}}|y-v|+m \frac{\gamma-\beta}{2(\delta+1)^{m}}|w-s| .
\end{aligned}
$$


A particular case can be obtained if $n=m=1, \alpha=0, \beta=2, \gamma=4, \delta=6$ and $\eta=8$. We get $f(x)=\varphi(x)=\frac{x}{7}+\frac{22}{7}, g(y)=\psi(y)=\frac{y}{8}+2, r=3, \bar{x}=\bar{y}=3$ and

$$
\begin{aligned}
S_{10} & =e\left(F(x, y) \cap B_{r}(\bar{x}), F(u, v)\right)+e\left(G(z, w) \cap B_{r}(\bar{y}), G(t, s)\right) \\
& \leq \frac{1}{8}|x-u|+\frac{1}{7}|y-v|+\frac{1}{8}|z-t|+\frac{1}{7}|w-s| .
\end{aligned}
$$

Example 2. Let us consider the space $\mathbb{R}^{2}$. Let us choose $0<\alpha_{i}<\beta_{i}<\gamma_{i}<\delta_{i}<\eta_{i}<$ $+\infty, n_{i}, m_{i} \in(0,1]$ for $i=1,2$, so that

$$
\max _{i=1,2}\left\{\frac{n_{i}\left(\gamma_{i}-\beta_{i}\right)}{2\left(\delta_{i}+1\right)^{n_{i}}}\right\}+\max _{i=1,2}\left\{\frac{m_{i}\left(\gamma_{i}-\beta_{i}\right)}{2\left(\delta_{i}+1\right)^{m_{i}}}\right\}<1
$$

and

$$
\max _{i=1,2}\left\{\frac{n_{i}\left(\gamma_{i}-\beta_{i}\right)}{2\left(\left(\eta_{i}+1\right)^{n_{i}}-\left(\alpha_{i}+1\right)^{n_{i}}\right)}\right\}+\max _{i=1,2}\left\{\frac{m_{i}\left(\gamma_{i}-\beta_{i}\right)}{2\left(\left(\eta_{i}+1\right)^{m_{i}}-\left(\alpha_{i}+1\right)^{m_{i}}\right)}\right\}<1 .
$$

Let us define the maps

$$
\begin{aligned}
& f_{i}:\left[0, \delta_{i}\right] \rightarrow\left[\frac{\beta_{i}+\gamma_{i}}{2}, \gamma_{i}\right], g_{i}:\left[\alpha_{i}, \eta_{i}\right] \rightarrow\left[\beta_{i}, \frac{\beta_{i}+\gamma_{i}}{2}\right], \\
& \varphi_{i}:\left[0, \delta_{i}\right] \rightarrow\left[\frac{\beta_{i}+\gamma_{i}}{2}, \gamma_{i}\right], \psi_{i}:\left[\alpha_{i}, \eta_{i}\right] \rightarrow\left[\beta_{i}, \frac{\beta_{i}+\gamma_{i}}{2}\right]
\end{aligned}
$$

for $i=1,2$ by

$$
\begin{aligned}
f_{i}(x) & =\frac{\gamma_{i}-\beta_{i}}{2\left(\delta_{i}+1\right)^{n_{i}}}(x+1)^{n_{i}}+\frac{\beta_{i}+\gamma_{i}}{2}, \\
g_{i}(x) & =C(x+1)^{n_{i}}+\beta_{i}-\left(\alpha_{i}+1\right)^{n_{i}} C \\
\varphi_{i}(x) & =\frac{\gamma_{i}-\beta_{i}}{2\left(\delta_{i}+1\right)^{m_{i}}}(x+1)^{m_{i}}+\frac{\beta_{i}+\gamma_{i}}{2} \\
\psi_{i}(x) & =D(x+1)^{m_{i}}+\beta_{i}-\left(\alpha_{i}+1\right)^{m_{i}} D
\end{aligned}
$$

where $C=\frac{\gamma_{i}-\beta_{i}}{2\left(\left(\eta_{i}+1\right)^{n_{i}}-\left(\alpha_{i}+1\right)^{n_{i}}\right)}$ and $D=\frac{\gamma_{i}-\beta_{i}}{2\left(\left(\eta_{i}+1\right)^{m_{i}}-\left(\alpha_{i}+1\right)^{m_{i}}\right)}$.

Let us denote $\bar{x}_{i}=\frac{\beta_{i}+\gamma_{i}}{2}$ and $\theta_{i}=\min \left\{\left|\delta_{i}-\bar{x}_{i}\right|,\left|\alpha_{i}-\bar{x}_{i}\right|\right\}$ for $i=1$, 2. Let us endow $\mathbb{R}^{2}$ with the metrics $\rho((x, y),(u, v))=\left(\left|\frac{x-u}{\theta_{1}}\right|^{p}+\left|\frac{y-v}{\theta_{2}}\right|^{p}\right)^{1 / p}, p \in(1,+\infty)$. Let us consider the sets $X_{i}=\left[0, \delta_{i}\right], Y_{i}=\left[\alpha_{i}, \eta_{i}\right]$ for $i=1,2$ and let $X=X_{1} \times X_{2}, Y=Y_{1} \times Y_{2}$. Let us define the multivalued maps $F: X \times Y \rightrightarrows X$ and $G: X \times Y \rightrightarrows Y$ by

$$
F\left(\left(x_{1}, x_{2}\right),\left(y_{1}, y_{2}\right)\right)=\left\{\left(\xi_{1}, \xi_{2}\right): g_{i}\left(y_{i}\right) \leq \xi_{i} \leq f_{i}\left(x_{i}\right)\right\}
$$

and

$$
G\left(\left(x_{1}, x_{2}\right),\left(y_{1}, y_{2}\right)\right)=\left\{\left(\xi_{1}, \xi_{2}\right): \psi_{i}\left(y_{i}\right) \leq \xi_{i} \leq \varphi_{i}\left(x_{i}\right)\right\} .
$$

We will check that the pair $(F, G)$ satisfies Theorem 1 .

Let us choose $r=2$ and $\bar{x}=\bar{y}=\left(\bar{x}_{1}, \bar{x}_{2}\right)$. By definition

$$
B_{r}(\bar{x}) \equiv B_{r}(\bar{y})=\left\{x=\left(x_{1}, x_{2}\right): \rho(x, \bar{x}) \leq 2\right\} .
$$

It is easy to see that $R_{2} \subseteq B_{2}(\bar{x}) \subseteq R_{1}$, where $R_{1}$ be the rectangular with vertices $\left(\alpha_{1}, \alpha_{2}\right),\left(\delta_{1}, \alpha_{2}\right),\left(\delta_{1}, \delta_{2}\right),\left(\alpha_{1}, \delta_{2}\right)$ and $R_{2}$ be the rectangular with vertices $\left(\beta_{1}, \beta_{2}\right),\left(\gamma_{1}, \beta_{2}\right)$, $\left(\gamma_{1}, \gamma_{2}\right),\left(\beta_{1}, \gamma_{2}\right)$ and $B_{2}(\bar{x})$ (Figure 2$)$ is an ellipse for $p=2$. 


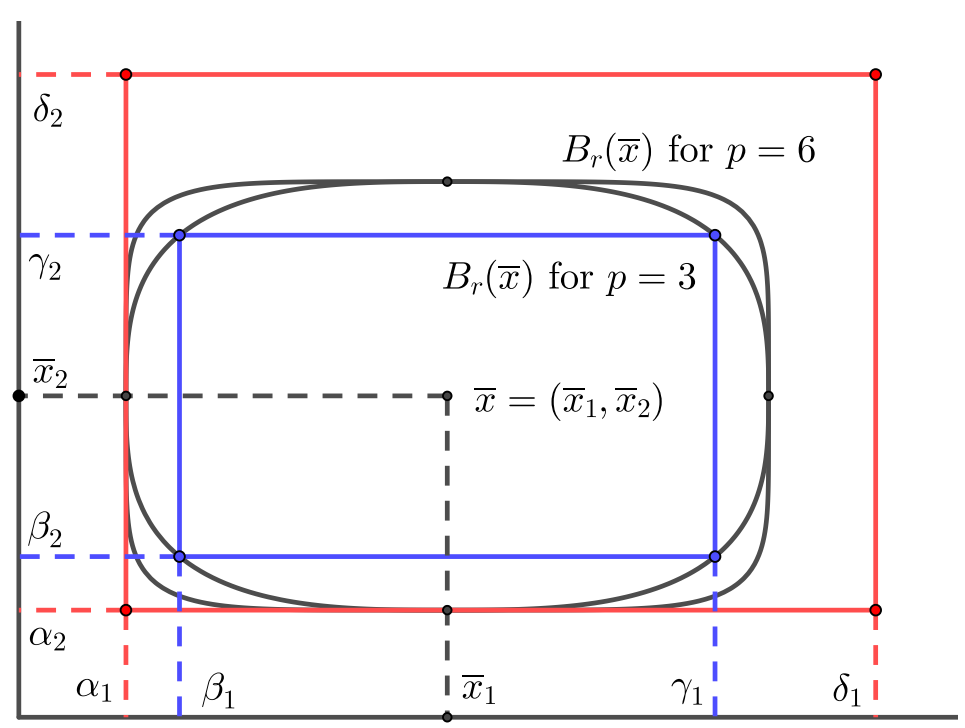

Figure 2. $R_{2} \subseteq B_{2}(\bar{x}) \subseteq R_{1}$.

Indeed, let $\left(x_{1}, x_{2}\right) \in R_{2}$. Then $\beta_{i} \leq x_{i} \leq \gamma_{i}$. Thereafter it holds for $i=1,2$

$$
\left|\frac{\beta_{i}+\gamma_{i}}{2}-x_{i}\right|=\left|\bar{x}_{i}-x_{i}\right| \leq \min \left\{\left|\delta_{i}-\bar{x}_{i}\right|,\left|\alpha_{i}-\bar{x}_{i}\right|\right\}=\theta_{i}
$$

and consequently we can write the inequalities

$$
\rho\left(\left(x_{1}, x_{2}\right),\left(\bar{x}_{1}, \bar{x}_{2}\right)\right)=\left(\left|\frac{\bar{x}_{1}-x_{1}}{\theta_{1}}\right|^{p}+\left|\frac{\bar{x}_{2}-x_{2}}{\theta_{2}}\right|^{p}\right)^{1 / p} \leq 2^{1 / p}<2 .
$$

From $g_{i}\left(\bar{x}_{i}\right) \leq \bar{x}_{i} \leq f_{i}\left(\bar{x}_{i}\right)$ it follows that $\bar{x}=\left(\bar{x}_{1}, \bar{x}_{2}\right) \in F\left(\bar{x}_{1}, \bar{x}_{2}\right)$ and from $\psi_{i}\left(\bar{x}_{i}\right) \leq$ $\bar{x}_{i} \leq \varphi_{i}\left(\bar{x}_{i}\right)$ it follows that $\bar{x}=\left(\bar{x}_{1}, \bar{x}_{2}\right) \in G\left(\bar{x}_{1}, \bar{x}_{2}\right)$ and therefore $d(\bar{x}, F(\bar{x}, \bar{y}))=d(\bar{y}, G(\bar{x}, \bar{y}))$ $=0 \leq r(1-\lambda)$ holds for any $r \geq 1$ and $\lambda \in[0,1)$.

We observe that there hold $F(x, y) \cap B_{r}(\bar{x})=F(x, y)$ and $G(x, y) \cap B_{r}(\bar{y})=G(x, y)$. Therefore we will need to calculate $e(F(x, y), F(u, v))$ and $e(G(z, w), G(t, s))$.

The set $F(x, y)=F\left(\left(x_{1}, x_{2}\right),\left(y_{1}, y_{2}\right)\right)$ is a rectangular with vertexes $\left(g\left(y_{1}\right), g\left(y_{2}\right)\right)$, $\left(f\left(x_{1}\right), g\left(y_{2}\right)\right),\left(f_{1}\left(x_{1}\right), f\left(x_{2}\right)\right)$ and $\left(g\left(y_{1}\right), f\left(x_{2}\right)\right)$. There are several possible cases: $g\left(y_{i}\right) \leq$ $g\left(v_{i}\right)$, or $g\left(v_{i}\right) \leq g\left(y_{i}\right)$ and $f\left(x_{i}\right) \leq f\left(u_{i}\right)$ or $f\left(u_{i}\right) \leq f\left(x_{i}\right)$ with all the possible combinations of $i=1,2$.

Let us first consider the case: $g\left(y_{1}\right) \leq g\left(v_{1}\right) \leq f\left(u_{1}\right) \leq f\left(x_{1}\right)$ and $g\left(y_{2}\right) \leq g\left(v_{2}\right) \leq$ $f\left(u_{2}\right) \leq f\left(x_{2}\right)$. It is easy to observe that $e(F(x, y), F(u, v))=\max \left\{A_{i}: i=1,2,3,4\right\}$ (Figure 3), where

$$
\begin{aligned}
& A_{1}=\operatorname{dist}\left(\left(g\left(y_{1}\right), g\left(y_{2}\right)\right), F(u, v)\right), \\
& A_{2}=\operatorname{dist}\left(\left(f\left(x_{1}\right), g\left(y_{2}\right)\right), F(u, v)\right), \\
& A_{3}=\operatorname{dist}\left(\left(f\left(x_{1}\right), f\left(x_{2}\right)\right), F(u, v)\right), \\
& A_{4}=\operatorname{dist}\left(\left(g\left(y_{1}\right), f\left(x_{2}\right)\right), F(u, v)\right) .
\end{aligned}
$$




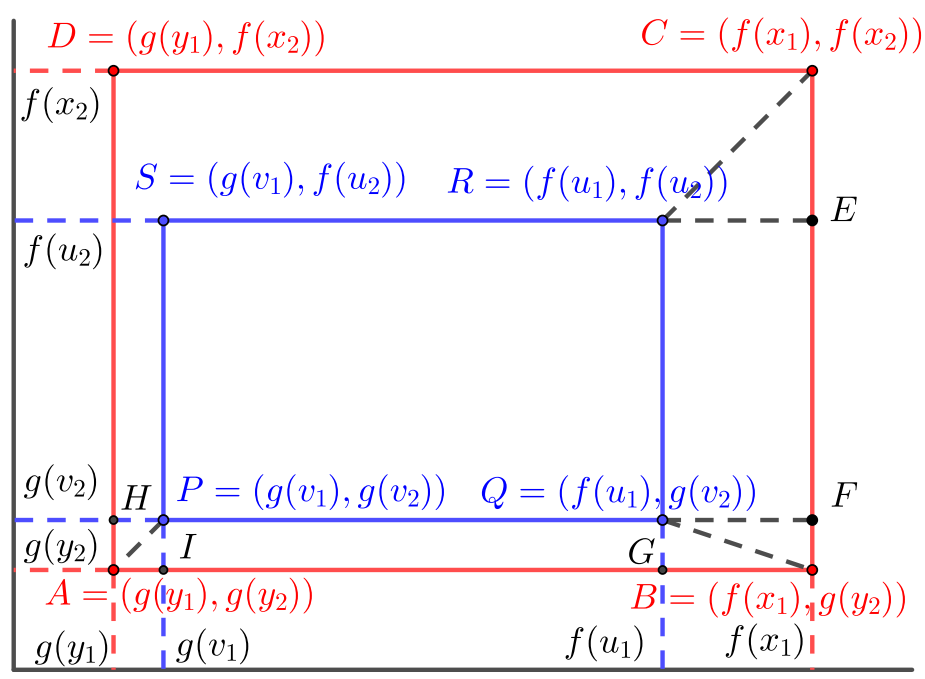

Figure 3. $e(F(x, y), F(u, v))=\max \left\{A_{i}: i=1,2,3,4\right\}$.

We will need the inequalities:

$$
\begin{gathered}
\left|f_{i}(x)-f_{i}(y)\right|=f_{i}^{\prime}(\zeta)|x-y| \leq n_{i} \frac{\gamma_{i}-\beta_{i}}{2\left(\delta_{i}+1\right)^{n_{i}}}|x-y|, \\
\left|g_{i}(x)-g_{i}(y)\right|=g_{i}^{\prime}(\zeta)|x-y| \leq n_{i} \frac{\gamma_{i}-\beta_{i}}{2\left(\left(\eta_{i}+1\right)^{n_{i}}-\left(\alpha_{i}+1\right)^{n_{i}}\right)}|x-y|,
\end{gathered}
$$

where $n_{i} \in(0,1)$ and

$$
f^{\prime}(\zeta) \leq n_{i} \frac{\gamma_{i}-\beta_{i}}{2\left(\delta_{i}+1\right)^{n_{i}}} \max \left\{(x+1)^{n_{i}-1}: x \in\left[0, \delta_{i}\right]\right\}=n_{i} \frac{\gamma_{i}-\beta_{i}}{2\left(\delta_{i}+1\right)^{n_{i}}}
$$

and

$$
\begin{aligned}
g_{i}^{\prime}(\zeta) & \leq n_{i} \frac{\gamma_{i}-\beta_{i}}{2\left(\left(\eta_{i}+1\right)^{n_{i}}-\left(\alpha_{i}+1\right)^{n_{i}}\right)} \max \left\{(x+1)^{n_{i}-1}: x \in\left[\alpha_{i}, \eta_{i}\right]\right\} \\
& =n_{i} \frac{\gamma_{i}-\beta_{i}}{2\left(\left(\eta_{i}+1\right)^{\left.n_{i}-\left(\alpha_{i}+1\right)^{n_{i}}\right)}\right.}=n_{i} C_{i} .
\end{aligned}
$$

We calculate

$$
\begin{aligned}
A_{1} & =\sqrt[p]{\left|\frac{g_{1}\left(v_{1}\right)-g_{1}\left(y_{1}\right)}{\theta_{1}}\right|^{p}+\left|\frac{g_{2}\left(v_{2}\right)-g_{2}\left(y_{2}\right)}{\theta_{2}}\right|^{p}} \\
& =\sqrt[p]{\left.\frac{1}{\theta_{1}^{p}}\left|n_{1} C_{1}\right|^{p}\left|v_{1}-y_{1}\right|^{p}+\frac{1}{\theta_{2}^{p}}\left|n_{2} C_{2}\right|^{p}\left|v_{2}-y_{2}\right|^{p}\right)} \\
& \leq\left(\max _{i=1,2}\left\{\frac{n_{i}\left(\gamma_{i}-\beta_{i}\right)}{2\left(\left(\eta_{i}+1\right)^{n_{i}}-\left(\alpha_{i}+1\right)^{n_{i}}\right)}\right\}\right) \rho\left(\left(v_{1}, v_{2}\right),\left(y_{1}, y_{2}\right)\right)
\end{aligned}
$$

and

$$
\begin{aligned}
A_{3} & =\sqrt[p]{\left|\frac{f_{1}\left(u_{1}\right)-f_{1}\left(x_{1}\right)}{\theta_{1}}\right|^{p}+\left|\frac{f_{2}\left(u_{2}\right)-f_{2}\left(x_{2}\right)}{\theta_{2}}\right|^{p}} \\
& =\sqrt[p]{\left.\frac{1}{\theta_{1}^{p}}\left|\frac{n_{1}\left(\gamma_{1}-\beta_{1}\right)}{2\left(\delta_{1}+1\right)^{n_{1}}}\right|^{p}\left|u_{1}-x_{1}\right|^{p}+\frac{1}{\theta_{2}^{p}}\left|\frac{n_{2}\left(\gamma_{2}-\beta_{2}\right)}{2\left(\delta_{2}+1\right)^{n_{2}}}\right|^{p}\left|u_{2}-x_{2}\right|^{p}\right)} \\
& \leq\left(\max _{i=1,2}\left\{\frac{n_{i}\left(\gamma_{i}-\beta_{i}\right)}{2\left(\delta_{i}+1\right)^{n_{i}}}\right\}\right) \rho\left(\left(x_{1}, x_{2}\right),\left(u_{1}, u_{2}\right)\right) .
\end{aligned}
$$


For the estimation of $A_{2}$ and $A_{4}$ let us denote (Figure 3)

$$
\begin{aligned}
& A=\left(g_{1}\left(y_{1}\right), g_{2}\left(y_{2}\right)\right), B=\left(f_{1}\left(x_{1}\right), g_{2}\left(y_{2}\right)\right), \\
& C=\left(f_{1}\left(x_{1}\right), f_{2}\left(x_{2}\right)\right), P=\left(g_{1}\left(v_{1}\right), g_{2}\left(v_{2}\right)\right), \\
& Q=\left(f_{1}\left(u_{1}\right), g_{2}\left(v_{2}\right)\right), R=\left(f_{1}\left(u_{1}\right), f_{2}\left(u_{2}\right)\right), \\
& I=\left(g_{1}\left(v_{1}\right), g_{2}\left(y_{2}\right)\right), G=\left(f_{1}\left(u_{1}\right), g_{2}\left(y_{2}\right)\right), \\
& F=\left(f_{1}\left(x_{1}\right), g_{2}\left(v_{2}\right)\right), E=\left(f_{1}\left(x_{1}\right), f_{2}\left(u_{2}\right)\right) .
\end{aligned}
$$

There holds

$$
\begin{aligned}
A_{2} & =\rho(B, Q) \leq \rho(G, Q)+\rho(Q, F)=\rho(P, I)+\rho(R, E) \\
& \leq \rho(A, P)+\rho(R, C) \\
& \leq\left(\max _{i=1,2}\left\{n_{i} C_{i}\right\}\right) \rho(v, y)+\left(\max _{i=1,2}\left\{\frac{n_{i}\left(\gamma_{i}-\beta_{i}\right)}{2\left(\delta_{i}+1\right)^{n_{i}}}\right\}\right) \rho(x, u) .
\end{aligned}
$$

By similar observation we can prove that

$$
A_{4} \leq\left(\max _{i=1,2}\left\{n_{i} C_{i}\right\}\right) \rho(v, y)+\left(\max _{i=1,2}\left\{\frac{n_{i}\left(\gamma_{i}-\beta_{i}\right)}{2\left(\delta_{i}+1\right)^{n_{i}}}\right\}\right) \rho(x, u) .
$$

Consequently $e(F(x, y), F(u, v) \leq \alpha \rho(x, u)+\beta \rho(y, v)$, where

$$
\alpha=\max _{i=1,2}\left\{\frac{n_{i}\left(\gamma_{i}-\beta_{i}\right)}{2\left(\delta_{i}+1\right)^{n_{i}}}\right\} \text { and } \beta=\max _{i=1,2}\left\{\frac{n_{i}\left(\gamma_{i}-\beta_{i}\right)}{2\left(\left(\eta_{i}+1\right)^{n_{i}}-\left(\alpha_{i}+1\right)^{n_{i}}\right)}\right\} .
$$

Let us denote the two rectangles $F(x, y)$ and $F(u, v)$ by $A B C D$ and $P Q R S$, respectively. We have just investigated the case $P Q R S \subseteq A B C D$. All the other cases are variants of Figure 4 and we can get that

$$
\begin{aligned}
e(F(x, y), F(u, v)) & \leq \max \{\rho(A, P), \rho(B, Q), \rho(C, R), \rho(D, S)\} \\
\leq & \alpha \rho(x, u)+\beta \rho(y, v) .
\end{aligned}
$$
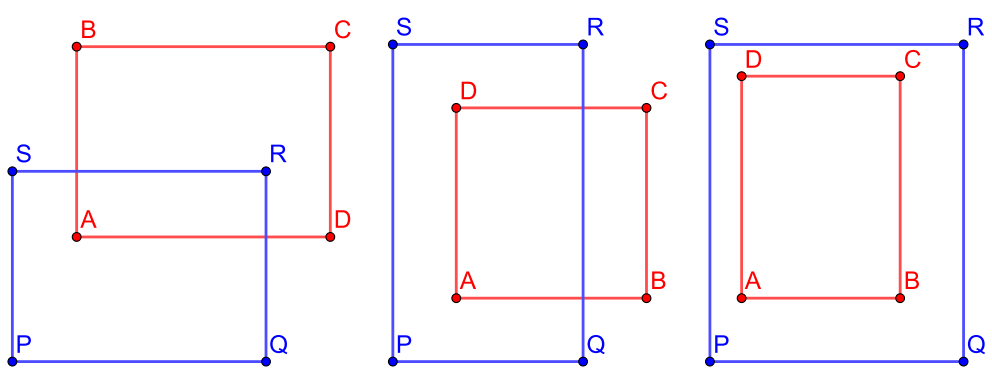

Figure 4. $A B C D$ and $P Q R S$.

By similar calculations for the multivalued map $G$ we get

$$
e(G(z, w), G(t, s)) \leq \gamma \rho(z, t)+\delta \rho_{p}(w, s)
$$

where

$$
\gamma=\max _{i=1,2}\left\{\frac{m_{i}\left(\gamma_{i}-\beta_{i}\right)}{2\left(\delta_{i}+1\right)^{m_{i}}}\right\} \text { and } \delta=\max _{i=1,2}\left\{\frac{m_{i}\left(\gamma_{i}-\beta_{i}\right)}{2\left(\left(\eta_{i}+1\right)^{m_{i}}-\left(\alpha_{i}+1\right)^{m_{i}}\right)}\right\}
$$

and thus

$$
e(F(x, y), F(u, v))+e(G(z, w), G(t, s)) \leq \alpha \rho(x, u)+\beta \rho(y, v)+\gamma \rho(z, t)+\delta \rho(w, s) .
$$

\subsection{Examples for the Existence of an Equilibrium in Oligopoly (Duopoly) Markets}

The theory of oligopoly (duopoly) markets was initiated in [21]. Following [22,23] we present the main features of an oligopoly model in economics. The oligopoly is a 
market structure in the presence of imperfect competition in which a limited number of large companies control the production and sale of a predominant part of the product in a particular sector of the economy. It is believed that oligopolies are the result of the trend in the economy towards concentration of capital and labor. The oligopoly is characterized by product differentiation; high barriers preventing the emergence of new "players"; limited access to information; non-price competition through advertising and other marketing activities, as well as price control. In the oligopolistic structure, large companies determine the behavior of competitors and take it into account when developing their strategy, which can be rivalry, even "trade wars" in terms of production volume, sales, and prices; the strategic interaction results in agreements (through secret or open collusion or without collusion) in order to guarantee stability and ensure high profits. They contribute to raising economic and organizational barriers, making it difficult for new "players" to emerge. This is the nature of the large initial capital costs for entering the business and achieving minimum effective production and sales capacity in view of economies of scale and resilience against competitors, the development of own research and development for product innovation, industrial and trade secrets. Oligopolistic market structures arise and are imposed by three key points (1) the concentration of assets; (2) inter-firm agreements; and (3) fencing off activities in order to gain market power, restrict competition, and generate large profits.

The distinctive feature of the oligopoly is that in determining individual supply and market price, companies are interdependent. The change in the market behavior of each of them can lead to a change in market conditions and possibly cause a change in the behavior of other companies.

The equilibrium quantity and price in the oligopoly will depend on the number of firms on the market, the information available, the strategy chosen by competitors and whether the firms in the market act independently of each other or in concert. The latter factor is the basis for two types of oligopolistic equilibrium-coherent and inconsistent, which differ significantly in end results and economic efficiency.

The classic model of uncoordinated oligopolistic behavior is the Cournot duopoly model, which considers the problem of the interdependence of firms in the market. The duopoly is a market structure in which two companies, protected from the emergence of other sellers, act as the only producers of standardized products that have no close substitutes, in which there are only two sellers of a particular product that are not interconnected by monopolistic agreement for prices market for selling products and quotas. The participants in the model try to maximize their payoff functions $\Pi_{1}(x, y)=x P(x+y)-C_{1}(x)$ and $\Pi_{2}(x, y)=y P(x+y)-C_{2}(x)$, respectively, where $P(Z)=P(x+y)$ be the inverse of the demand function and $C_{1}(x)$ and $C_{2}(y)$ be the cost functions of the two players. By maximizing its payoff functions, the players get their response functions $F: X \times Y \rightarrow X$ and $f: X \times Y \rightarrow X$, respectively.

The company equilibrium would become market if the supply of one company is equal to the supply of the other company, so that none of the companies is motivated to change their positions condition for market equilibrium. This condition is present if each of the companies produces one third of the total market supply under conditions of perfect competition and both companies sell at a specific market price, which is one third of the market price. Taking into account the strategic considerations of the companies, their behavior will depend on the decisions of their competitors.

Cournot's theory is based on competition and the fact that buyers announce prices and sellers adjust their products to those prices. Each company evaluates the product search function and then sets the quantity that will be sold, assuming that the competitor's output remains constant.

Deeper research on the oligopoly market can be found in [22-25].

Cournot's classical model deals with a maximization of the payoff functions of each of the players. A different approach is presented in [18], where attention is paid to the response functions of the players. The benefits of this approach are commented on in [18]. 
We will just say that as far as the players do not have a perfect knowledge of the market they react in some sense by not maximizing their payoff function, but rather by choosing a strategy based on their production and their rival's production levels. The solution of the maximization of the payoff function is actually the coupled fixed points $(x, y)$, such that $x=F(x, y)$ and $y=f(x, y)$.

Focusing on response functions allows us to put Cournot and Bertand's models together. Indeed let the first company reaction be $F(X, Y)$ and the second one $f(X, Y)$, where $X=(x, p)$ and $Y=(y, q)$. Here $x$ and $y$ denote the output quantity and $(p, q)$ are the prices set by players. In this, companies can compete in terms of both price and quantity.

A disadvantage of the presented model is that players do not choose a fixed production of a fixed price. Actually, the response of each player is any quality from a set of possible productions or a price from possible prices. Therefore we will consider the response functions $F: X \times Y \rightrightarrows U \subset X$ and $f: X \times Y \rightrightarrows V \subset Y$ be multivalued maps and a market equilibrium will be the pair $(x, y)$, such that $x \in F(x, y)$ and $y \in f(x, y)$.

Now we can restate Theorem 1 in terms of oligopoly.

Theorem 2. Let us assume that two companies are offering products that are perfect substitutes. The first one can produce qualities from the set $X$ and the second firm can produce qualities from the set $Y$, where $X$ and $Y$ be nonempty subsets of a partially ordered complete metric space $(Z, \rho)$ and $\bar{x} \in X, \bar{y} \in Y$. Consider $F: X \times Y \rightrightarrows X$ and $G: X \times Y \rightrightarrows Y$ to be the response function of players one and two, respectively. Let $F$ and $G$ satisfy all the conditions in Theorem 1.

Then there exists at least one market equilibrium point $(x, y) \in B_{r}(\bar{x}) \times B_{r}(\bar{y})$, which is a coupled fixed point for the ordered pair of response functions $(F, G)$.

Example 3: Let us consider in Example 1 two firms, producing one commodity, which is a perfect substitute. Let us put $\alpha=10, \beta=30, \gamma=50, \delta=80$ and $\eta=100$ in Example 1. We may consider the interval $[0, \eta]$ as the set of the total production. Let the first firm be a smaller one and its production set is $[0, \delta]$ and the second one be a larger firm with a production set $[\alpha, \eta]$. Let $n=1$ and $m=1 / 2$. Then for any initial start $[x, y]$ in the market the first firm chooses a production from the set

$$
\left[\frac{y}{9}+\frac{260}{9}, \frac{10}{81} x+\frac{3250}{81}\right]
$$

and the second firm from the set

$$
\left[\frac{10 \sqrt[2]{y+1}+30 \sqrt[2]{101}-40 \sqrt[2]{11}}{\sqrt[2]{101}-\sqrt[2]{11}}, \frac{10}{9} \sqrt[2]{x+1}+40\right]
$$

and

$$
\begin{aligned}
S_{11} & =e\left(F(x, y) \cap B_{r}(\bar{x}), F(u, v)\right)+e\left(G(z, w) \cap B_{r}(\bar{y}), G(t, s)\right) \\
& \leq \frac{10}{81}|x-u|+\frac{1}{9}|y-v|+\frac{5}{9}|z-t|+\gamma|w-s|,
\end{aligned}
$$

where $\gamma=\frac{5}{\sqrt{101}-\sqrt{11}}<\frac{5}{6}$. From $\max \left\{\frac{10}{81}+\frac{5}{9}, \frac{1}{9}+\frac{5}{6}\right\}=\max \left\{\frac{55}{81}, \frac{17}{18}\right\}=\frac{17}{18}<1$ it follows that the pair of response functions satisfies Theorem 2 and consequently there exists an equilibrium pair of productions $(x, y)$, such that $x \in F(x, y)$ and $y \in G(x, y)$.

Example 4. Let us consider a model of a duopoly with two players, producing one good, which is a complete substitute, and let they compete on qualities and prices simultaneously. Let us choose in Example 2, $\alpha_{1}=10, \beta_{1}=30, \gamma_{1}=40, \delta_{1}=60, \eta_{1}=100$, $\alpha_{2}=1, \beta_{2}=3, \gamma_{2}=4, \delta_{2}=5, \eta_{2}=8 n_{1}=1, n_{2}=1 / 2, m_{1}=1 / 2, m_{2}=1 / 4$. Let us consider the sets $X_{i}=\left[0, \delta_{i}\right], Y_{i}=\left[\alpha_{i}, \eta_{i}\right]$ for $i=1,2$ and let $X=X_{1} \times X_{2}, Y=Y_{1} \times Y_{2}$ and the multivalued maps $F: X \times Y \rightrightarrows X$ and $G: X \times Y \rightrightarrows Y$ from Example 2, which are the response functions of the two players, respectively, where the first coordinates are the response on the qualities and the second coordinate is the response on the price. Let 
us endow $\mathbb{R}^{2}$ with the metrics $\rho((x, y),(u, v))=\left(\left|\frac{x-u}{\theta_{1}}\right|^{p}+\left|\frac{y-v}{\theta_{2}}\right|^{p}\right)^{1 / p}, p \in(1,+\infty)$ from Example 2.

We get that

$$
\begin{aligned}
S_{12} & =e(F(x, y), F(u, v))+e(G(z, w), G(t, s)) \\
& \leq 0.2 \rho(x, u)+0.4 \rho(y, v)+0.2 \rho(z, t)+0.4 \rho(w, s)
\end{aligned}
$$

From the inequality $\max \{0.2+0.2,0.4+0.4\}<1$ it follows that we can apply Theorem 2. Consequently there exists an equilibrium pair of productions and prices $((x, p),(y, q))$, such that $(x, p) \in F((x, p),(y, q))$ and $(y, q) \in G((x, p),(y, q))$. The actual values of $\alpha, \beta, \gamma$ and $\delta$ are smaller.

\subsection{Example for the Existence of an Equilibrium in Ecology}

Despite the long history of aquatic ecosystems contamination and numerous extensive research undertaken, there are still open questions that remain to be explored. Revealing the relationship between the pollutant, pathway (water) and biota will help water bodies assessment and management. Heavy metals and other contaminants can bioaccumulate in aquatic organisms depending on their bioavailability and concentration in the water media. Among the most applied biomonitors for evaluating sources and releases of contaminants are aquatic bryophytes. Many studies reported a positive correlation between contaminants in aqueous environment and in mosses, for example for $\mathrm{Cu}$ [26]. Nevertheless, numerous research have reported that aquatic mosses often accumulate toxic elements in concentrations much higher than those reached in their ambient water media [27] or even when the contaminant in water samples is below the LOD [28].

Now we can restate Theorem 1 in terms of ecology.

Theorem 3. Let us assume that in an aquatic ecosystem there is one pollutant and a kind of aquatic organisms that accumulate the pollutant. The pollutant can have qualities from the set $Y$ and the aquatic organisms can accumulate qualities from the pollutant from the set $X$, where $X$ and $Y$ be nonempty subsets of a partially ordered complete metric space $(Z, \rho)$ and $\bar{x} \in X, \bar{y} \in Y$. Consider $F: X \times Y \rightrightarrows X$ and $G: X \times Y \rightrightarrows Y$ to be the response function of the aquatic organisms and the pollutant, respectively. Let $F$ and $G$ satisfy all the conditions in Theorem 1.

Then there exists at least one point $(x, y) \in B_{r}(\bar{x}) \times B_{r}(\bar{y})$, which is a generalized coupled fixed point for the ordered pair of response functions $(F, G)$.

Example 5. Let us consider in Example 1 two media (water and biota), the first one of which is an aquatic ecosystem (e.g. river water), which is polluted continuously and the second one is a bryophyte species that accumulates the contaminant. Let the pollution be from the set $Y=[\alpha, \eta]$ and the accumulated substance in the bryophyte be from the set $X=[0, \delta]$. Let us consider Example 1 with $\alpha=1, \beta=3, \gamma=7, \delta=8, \eta=10, n=3 / 4$ and $m=4 / 5$. If the pollution is $y$ and the accumulated substance in the bryophyte is estimated as $x$, then, due to the new inflow of pollution and the accumulation of the substance in the bryophyte, which are also reproduced, the pollution and the accumulation change in time to be the multivalued maps

$$
F \rightrightarrows[g(y), f(x)] \text { for all }(x, y) \in X \times Y
$$

and

$$
G \rightrightarrows[\psi(y), \varphi(x)] \text { for all }(x, y) \in X \times Y
$$

respectively, where

$$
f(x)=\frac{2}{\sqrt[4]{9^{3}}} \sqrt[4]{(x+1)^{3}}+5, g(y)=\frac{2}{\sqrt[4]{11^{3}}-\sqrt[4]{2^{3}}} \sqrt[4]{(y+1)^{3}}+3-\frac{2 \sqrt[4]{2^{3}}}{\sqrt[4]{11^{3}}-\sqrt[4]{2^{3}}}
$$


and

$$
\varphi(x)=\frac{2}{\sqrt[5]{9^{4}}} \sqrt[4]{(x+1)^{5}}+5, \quad \psi(y)=\frac{2}{\sqrt[5]{11^{4}}-\sqrt[5]{2^{4}}} \sqrt[5]{(y+1)^{4}}+3-\frac{2 \sqrt[5]{2^{4}}}{\sqrt[5]{11^{4}}-\sqrt[5]{2^{4}}} .
$$

From Example 1 we get the inequality

$$
\begin{aligned}
S_{13} & =e(F(x, y), F(u, v))+e(G(z, w), G(t, s)) \\
& \leq 0.3 \rho(x, u)+0.4 \rho(y, v)+0.3 \rho(z, t)+0.4 \rho(w, s) .
\end{aligned}
$$

and consequently there exists an equilibrium pair of $(x, y)$, such that $x \in F(x, y)$ and $y \in G(x, y)$. The actual values of $\alpha, \beta, \gamma$ and $\delta$ are smaller.

Author Contributions: The authors are listed in an alphabetical order. All authors have read and agreed to the published version of the manuscript.

Funding: M.H. is partially supported by Shumen University Grant Number Rd-08-42/ 2021, and D.N. is partially supported by the Bulgarian National Fund for Scientific Research Grant Number KP-06-H22/4.

Institutional Review Board Statement: Not applicable.

Informed Consent Statement: Not applicable.

Data Availability Statement: Not applicable.

Acknowledgments: The authors would like to thank the anonymous reviewers for their comments and recommendations that have improved the value of the article.

Conflicts of Interest: The authors declare no conflict of interest.

\section{References}

1. Nadler, S.B. Multi-Valued Contraction Mappings. Pac. J. Math. 1969, 30, 475-488. [CrossRef]

2. Dontchev, A.; Hager, W. An inverse mapping theorem for set-valued maps. Proc. Am. Math. Soc. 1994, 121, 481-489. [CrossRef]

3. Ahmad, W.; Sarwar, M.; Abdeljawad, T.; Rahmat, G. Multi-Valued Versions of Nadler, Banach, Branciari and Reich Fixed Point Theorems in Double Controlled Metric Type Spaces with Applications. AIMS Math. 2021, 6, 477-499. [CrossRef]

4. Alsaedi, A.; Broom, A.; Ntouyas, S.K.; Ahmad, B. Nonlocal Fractional Boundary Value Problems Involving Mixed Right and Left Fractional Derivatives and Integrals. Axioms 2020, 9, 50. [CrossRef]

5. Chen, L.; Yang, N.; Zhou, J. Common Attractive Points of Generalized Hybrid Multi-Valued Mappings and Applications. Mathematics 2020, 8, 1307. [CrossRef]

6. Shoaib, M.; Sarwar, M.; Kumam, P. Multi-Valued Fixed Point Theorem via F-Contraction of Nadler Type and Application to Functional and Integral Equations. Bol. Soc. Paran. Mat. 2021, 39, 83-95. [CrossRef]

7. Bhaskar, T.G.; Lakshmikantham, N. Fixed Point Theorems in Partially Ordered Metric Spaces and Applications. Nonlinear Anal. 2006, 65, 1379-1393. [CrossRef]

8. George, R.; Mitrović, Z.D.; Radenovixcx, S. On Some Coupled Fixed Points of Generalized T-Contraction Mappings in a $b_{v}(s)-$ Metric Space and Its Application. Axioms 2020, 9, 129. [CrossRef]

9. Kim, K.S. A Constructive Scheme for a Common Coupled Fixed Point Problems in Hilbert Space. Mathematics 2020, 8, 1717. [CrossRef]

10. Kishore, G.; Rao, B.S.; Radenović, S.; Huang, H.P. Caristi Type Cyclic Contraction and Coupled Fixed Point Results in Bipolar Metric Spaces. Sahand Commun. Math. Anal. 2020, 17, 1-22. [CrossRef]

11. Shateri, T. Coupled fixed points theorems for non-linear contractions in partially ordered modular spaces. Int. J. Nonlinear Anal. Appl. 2020, 11, 133-147. [CrossRef]

12. Kirk, W.; Srinivasan, P.; Veeramani, P. Fixed points for mappings satisfying cyclical contractive conditions. Fixed Point Theory 2003, 4, 79-189.

13. Eldred, A.; Veeramani, P. Existence and convergence of best proximity points. J. Math. Anal. Appl. 2006, 323, 1001-1006. [CrossRef]

14. Abdeljawad, T.; Ullah, K.; Ahmad, J.; Sen, M.D.L.; Ulhaq, A. Approximation of Fixed Points and Best Proximity Points of Relatively Nonexpansive Mappings. J. Math. 2020, 2020, 8821553. [CrossRef]

15. Debnath, P.; Srivastava, H.M. Global Optimization and Common Best Proximity Points for Some Multivalued Contractive Pairs of Mappings. Axioms 2020, 9, 102. [CrossRef]

16. Mishra, L.N.; Dewangan, V.; Mishra, V.N.; Karateke, S. Best proximity points of admissible almost generalized weakly contractive mappings with rational expressions on $b$-metric spaces. J. Math. Comput. Sci. 2021, 22, 97-109. [CrossRef] 
17. Pant, R.; Shukla, R.; Rakocevic, V. Approximating best proximity points for Reich type non-self nonexpansive mappings. Rev. R. Acad. Cienc. Exactas Fìs. Nat. Ser. A Mat. RACSAM 2020, 114, 197. [CrossRef]

18. Dzhabarova, Y.; Kabaivanov, S.; Ruseva, M.; Zlatanov, B. Existence, Uniqueness and Stability of Market Equilibrium in Oligopoly Markets. Adm. Sci. 2020, 10, 70. [CrossRef]

19. Kabaivanov, S.; Zlatanov, B. A variational principle, coupled fixed points and market equilibrium. Nonlinear Anal. Model. Control. 2021, 26, 169-185. [CrossRef]

20. Zhang, X. Fixed Point Theorems of Multivalued Monotone Mappings in Ordered Metric Spaces. Appl. Math. Lett. 2010, 23, 235-240. [CrossRef]

21. Cournot, A.A. Researches into the Mathematical Principles of the Theory of Wealth, Translation ed.; Macmillan: New York, NY, USA, 1897.

22. Friedman, J.W. Oligopoly Theory; Cambradge University Press: Cambradge, UK, 2007.

23. Matsumoto, A.; Szidarovszky, F. Dynamic Oligopolies with Time Delays; Springer Nature Singapore Pte Ltd.: Singapore, 2018.

24. Okuguchi, K.; Szidarovszky, F. The Theory of Oligopoly with Multi-Product Firms; Springer: Berlin/Heidelberg, Germany, 1990.

25. Smith, A. A Mathematical Introduction to Economics; Basil Blackwell Limited: Oxford, UK, 1982.

26. Empain, A.M. A posteriori detection of heavy metal pollution of aquatic habitats. In Methods in Bryology. Proc. Bryol. Method. Workshop, Mainz; Glime, J.M., Ed.; Hattori Bot Lab.: Nichinan, Japan, 1988; pp. 213-220.

27. Gecheva, G.; Yurukova, L. Water pollutant monitoring with aquatic bryophytes: A review. Environ. Chem. Lett. 2014, 12, 49-61. [CrossRef]

28. Gecheva, G.; Yancheva, V.; Velcheva, I.; Georgieva, E.; Stoyanova, S.; Arnaudova, D.; Stefanova, V.; Georgieva, D.; Genina, V.; Todorova, B.; et al. Integrated Monitoring with Moss-Bag and Mussel Transplants in Reservoirs. Water 2020, 12, 1800. [CrossRef] 